\title{
Dynamic Modeling and Uncertainties Analysis for Space Station Redocking
}

\author{
Rui Chen ${ }^{1, a}$, Shengjing Tang ${ }^{1, b}$, Xuzhong $\mathrm{Wu}^{2, \mathrm{c}}$ and Guojiang Sun ${ }^{3}$ \\ ${ }^{1}$ Beijing Institute of Technology, Beijing 100081, China \\ ${ }^{2}$ Mechanical and Electrical Overall Design Dept., 100854, China \\ ${ }^{3}$ China Academy of Space Technology, 100094, China \\ achenrui_bit@163.com, btangsj@bit.edu.cn, cale10@vip.qq.com
}

Keywords: Space Station, Redocking, Uncertainty, generalized Polynomial Chaos.

\begin{abstract}
Space station redocking is a long period process which suffers uncertainties from external environment and space station itself. Dynamic model of redocking is derived firstly, then generalized Polynomial Chaos (gPC) method is used to analysis the influence on attitude motion caused by uncertainties. Numerical simulation show that if redocking begins from "Zero State", then uncertainties cause great impact on the process.
\end{abstract}

\section{Introduction}

The technology of redocking is one of the key problems for space station on-orbit assembly. Redocking is defined as transferring the docked cabin from the axial port to the lateral port for the benefit of next docking. Since the configuration and inertia of space station would change dramatically during redocking, so dynamic modeling and analysis of the system become critical. Although the existing works [1-3] have researched on the dynamic models in terms of redocking, but they didn't take the uncertain factors of redocking into consideration.

However, the uncertainty factors can seriously impact the work of space station. These factors are in the result of the special environment of the space and the structure of the space station. It is worth exploring the influence of uncertain factors in the process of redocking. In the recent years, generalized Polynomial Chaos (gPC), a spectrum analysis method, is popular in the research of uncertainty analysis. For a smooth function model, gPC can even realize spectrum convergence [4].

This paper firstly establish dynamic model of redocking, then introduces the basic principles of gPC and especially its algorithm of stochastic collocation method, and studies on the impact of the uncertain factors upon attitude angles in the process of redocking.

\section{Redocking model of space station}

Space station is a complex rigid-flexible coupling multibody system which mainly composed of core cabin, node cabin, several test cabins, manipulator and several solar arrays. This paper explores how the uncertain factors affect the attitude of space station when it redocks, to facilitate the research, the original system is simplified as a two rigid body model consisting core cabin and node cabin (since the two cabins are connected rigidly, they are together marked as A) and a test cabin (marked as B). This section uses Kane method to model the simplified system.

\subsection{Coordinate systems.}

In the process of modeling, four orthogonal coordinate system are introduced: the geocentric inertial coordinate frame $X_{E} Y_{E} Z_{E}$, the orbital coordinate frame $X_{O} Y_{O} Z_{O}$, the body coordinate frame of $A X_{A} Y_{A} Z_{A}$ and $B X_{B} Y_{B} Z_{B}$, and the entire system coordinate frame $X_{S} Y_{S} Z_{S}$. The unit vectors for each coordinate frame are $\mathbf{e}_{i}, \mathbf{o}_{1}, \mathbf{a}_{i}, \mathbf{b}_{i}, \mathbf{s}(i=1,2,3)$ respectively. 


\subsection{Attitude Coordinate.}

In this paper, the angles $q_{1}, q_{2}, q_{3}$ are used to describe the orientation of $X_{s} Y_{s} Z_{s}$ relative to $X_{O} Y_{O} Z_{O}$, and they are named as pitch angle, roll angle, and yaw angle respectively, and the rotation sequence is Body213. The attitude angle of $\mathrm{B}$ relative to $\mathrm{A}$ is $a, b$ and the rotation sequence is Body 23.

\subsection{Generalized speed.}

According to orbital mechanics, if the distance from the geocentre to the space station's C.M. is R, then the absolute velocity of the space station's with respect to the geocentre is ${ }^{E} \boldsymbol{V}^{S}=\sqrt{\mathrm{m} / R} \mathbf{o}_{1}$, and the angular velocity of the orbital coordinate frame with respect to the inertia coordinate is ${ }^{\mathrm{E}} \boldsymbol{w}^{0}=\sqrt{m / \mathrm{R}^{3}} \mathbf{o}_{2}$, in which $m$ refers to the Earth gravitational constant.

If the angular velocity of $X_{s} Y_{s} Z_{s}$ with respect to $X_{0} Y_{0} Z_{0}$ is ${ }^{\circ} w^{s}$, assuming that the system generalized speed $u_{i}(i=1,2,3)$ is the coordinate component of ${ }^{\circ} w^{s}$ expressed in $X_{\circ} Y_{0} Z_{0}$, i.e. ${ }^{\circ} \boldsymbol{w}^{\mathrm{s}}=u_{1} \mathbf{o}_{1}+u_{2} \mathbf{O}_{2}+u_{3} \mathbf{O}_{3}$. In accordance to the superposition principle of angular velocity, the absolute angular velocity of $X_{S} Y_{S} Z_{S}$ with respect to $X_{E} Y_{E} Z_{E}$ is ${ }^{E} \boldsymbol{w}^{s}={ }^{E} \boldsymbol{w}^{0}+{ }^{\circ} \boldsymbol{w}^{s}$.

Based on the conversion relation between orbital frame and the system frame, the kinematic equation of the system is:

$$
\begin{aligned}
& \text { \& }=\left(u_{1} s_{3}+u_{2} c_{3}\right) / c_{2} \\
& \text { \& }=u_{1} c_{3}-u_{2} s_{3} \\
& \text { \& }=\left(u_{1} s_{3}+u_{2} c_{3}\right) s_{2} / c_{2}+u_{3}
\end{aligned}
$$

In which $s_{i}, c_{i}$ refers to $\sin q_{1}, \cos q_{i}$, respectively.

The absolute velocities of the center of mass of $A$ and $B$ are ${ }^{E} \boldsymbol{v}^{A}$ and ${ }^{E} \boldsymbol{v}^{B}$, and their absolute angular velocities are ${ }^{E} \boldsymbol{w}^{A}$ and ${ }^{E} \boldsymbol{w}^{B}$, respectively, which can be derived on the basis of the velocity of the systematic center of mass ${ }^{E} \boldsymbol{v}^{S}$, the angular velocity of the system ${ }^{\mathrm{E}} \boldsymbol{w}^{\mathrm{S}}$ and the geometric relationships within the system. The detail derivation is complex and tedious, it is omitted here but can be referred to the literature[5]. The partial velocities and partial angular velocities of A and B can be derived as:

$$
\boldsymbol{v}_{\mathrm{r}}^{\mathrm{k}}=\frac{\text { 扯 } \boldsymbol{v}^{\mathrm{K}}}{\text { 抖 } \boldsymbol{r}_{\mathrm{r}}}, \quad \boldsymbol{w}_{\mathrm{r}}^{\mathrm{k}}=\frac{{ }^{\mathrm{E}} \boldsymbol{w}^{\mathrm{K}}}{u_{\mathrm{r}}}
$$

In which $K=A, B, r=1,2,3$.

\subsection{Generalized force.}

Considering the control moment of A to B as the internal force within the system, then the external forces to the system are gravity $\boldsymbol{F}_{\mathrm{g}}$ and gravitational gradient torque $\boldsymbol{T}_{\mathrm{g}}$ :

$$
\boldsymbol{F}_{\mathrm{g}}^{\mathrm{K}}=-\frac{m m_{\mathrm{K}}}{\left|\boldsymbol{R}_{\mathrm{K}}\right|^{3}} \boldsymbol{R}_{\mathrm{K}}, \quad \boldsymbol{T}_{\mathrm{g}}^{\mathrm{K}}=\frac{3 m}{\left|\boldsymbol{R}_{\mathrm{K}}\right|^{5}} \boldsymbol{R}_{\mathrm{K}} \quad \boldsymbol{J}_{\mathrm{K}} \quad \boldsymbol{R}_{\mathrm{K}} \text { 酉 }
$$

In which $\boldsymbol{R}_{\mathrm{K}}$ is the position vector from the geocenter to K body's center of mass, $m_{\mathrm{K}}$ is the mass of $\mathrm{K}$, and $\boldsymbol{J}_{\mathrm{K}}$ is the inertia dyad of $\mathrm{K}$ relative to the center of mass.

Generalized active force $F_{r}$ and generalized inertia force $F_{r}^{*}$ are defined as:

$$
\begin{aligned}
& F_{\mathrm{r}}=\stackrel{\circ}{a}\left(\begin{array}{lll}
v_{\mathrm{r}}^{\mathrm{k}} & ? \boldsymbol{F}_{\mathrm{g}}^{\mathrm{K}} & \boldsymbol{w}_{\mathrm{r}}^{\mathrm{K}} ? \boldsymbol{T}_{\mathrm{g}}^{\mathrm{K}}
\end{array}\right)
\end{aligned}
$$

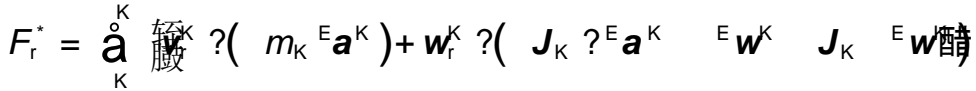

In which ${ }^{\mathrm{E}} \mathbf{a}^{\mathrm{K}}$ is the absolute acceleration of K's center of mass, ${ }^{\mathrm{E}} \mathbf{a}^{\mathrm{K}}$ is the absolute angular acceleration of $\mathrm{K}$.

\subsection{System state equation.}

As Kane's equation reveals, the sum of generalized active force and generalized inertia force is equal to 0:

$$
F_{\mathrm{r}}+F_{\mathrm{r}}^{*}=0(\mathrm{r}=1,2,3)
$$


The attitude kinematic equation (1) and the attitude dynamic equation (5) constitute the system governing equation and can be combined into one equation:

$$
X^{\&}=f(t, X, P)
$$

In which $\boldsymbol{X}=\left[\boldsymbol{q}^{\top}, \boldsymbol{u}^{\top}\right]^{\top} \boldsymbol{?} \mathbf{i}^{6}$, and $\boldsymbol{P}$ refers to some other system parameters. If the initial state $\boldsymbol{X}_{0}$ and the system's parameter $\boldsymbol{P}$ are known, then the state variable $\boldsymbol{X}$ at any moment $t$ can be calculated. As a result, the equation (6) can be seen as the deterministic model of the system.

\subsection{System's uncertainty model.}

In a real system, some parameters usually don't equal to nominal values exactly but only random values around it. Since it is impossible to measure the precise values, these parameters are defined as uncertainty parameters of the system. As a result, the system parameter $\boldsymbol{P}$ can be divided into uncertainty parameters $\boldsymbol{Z}$ and certainty parameters $\boldsymbol{D}$. Then the system equation (6) can be transformed to be:

$$
X^{\&}=f(t, X, Z, D)
$$

As the certainty parameter $\boldsymbol{D}$ is already known so that the equation (7) can be simplified into:

$$
x^{\&}=f(t, X, Z)
$$

In the equation (8), the value of the state variable $\boldsymbol{X}$ at the moment $t$ is relative to real values of $\boldsymbol{Z}$. The uncertainty of $\boldsymbol{Z}$ decides the uncertainty of $\boldsymbol{X}$, which can be described as $\boldsymbol{X}=\boldsymbol{X}(t, \boldsymbol{Z})$. So equation (9) is called uncertain model of the system.

Suppose the observation needs researching is $Y$, which is a value that can be deduced from the system equation, i.e., $\boldsymbol{Y}=\boldsymbol{Y}(t, \boldsymbol{X}, \boldsymbol{Z}) . \boldsymbol{Y}$ can be a scalar or a vector. Normally, the statistical information of $\boldsymbol{Y}$ in the effect of $\boldsymbol{Z}$ is expected to be researched. Among the information, the average value $\overline{\boldsymbol{Y}}$ and the variance $\boldsymbol{s}$ (or standard deviation $\boldsymbol{s}$ ) are seen as the most important. To solve the problem, the next section will explore how to use gPC to study the probability distribution of $\boldsymbol{Y}$.

\section{The method of gPC}

gPC is essentially a spectrum method which means to have a random quantity or a random process expand orthogonally on the gPC basis function. This section introduces the gPC basis function at first and then discusses how to process gPC expansion.

\section{1 gPC basis function}

For a random variable $Z$, Nth-order gPC basis function $\left\{f_{k}(Z)\right\}_{k=0}^{N}$ is a orthogonal set consist of polynomials from order 0 to order $N$, in which the kth term $f_{k}(Z)$ is a polynomial scalar function of $Z$ of kth-order, The orthogonal conditions of gPC basis function are defined as:

$$
\mathrm{E} \text { 轸 }(z) \mathrm{f}_{\mathrm{k}}(z)=g_{\mathrm{j}} d_{\mathrm{j} k}, \mathrm{j}, \mathrm{k} \text { ? } ¥
$$

In which $E[g]$ means the expectation of the expressions in bracket, $g_{j}=E\left[f_{j}^{2}(Z)\right] ? \mathbf{i}$ is the normalization factors, $d_{j k} \hat{\imath} \mathbf{i}$ is the Kronecker function, and $¥$ is non-negative integer set.

If $\boldsymbol{Z}=\left[Z_{1}, \mathrm{~L}, Z_{\mathrm{d}}\right]$ is a d-dimensional random vector and $Z_{\mathrm{i}}(\mathrm{i}=1, \mathrm{~L}, \mathrm{~d})$ are the independent and identically distributed random variables, then d-dimensional Nth-order gPC basis function $\left\{F_{k}(z)\right\}_{|k|=0}^{N}$ is defined as a orthogonal set consist of polynomials from order 0 to order $N$, in which the $\mathrm{k}$-th term $\mathrm{F}_{\mathrm{k}}(\boldsymbol{z})$ is a d-dimensional polynomial vector function of degree $|\mathbf{k}|$ by multiple multiplication of the single variable gPC:

$$
\mathrm{F}_{\mathrm{k}}(\boldsymbol{Z})=\mathrm{f}_{\mathrm{k}_{1}}\left(Z_{1}\right) \mathrm{k}_{2}\left(Z_{2}\right) \mathrm{L} \quad \mathrm{k}_{\mathrm{d}}\left(Z_{\mathrm{C}} \mathrm{C}\right. \text { 會 }
$$

In which subscript $\mathbf{k}=\left(\mathrm{k}_{1}, \mathrm{~L}, \mathrm{k}_{\mathrm{d}}\right)$ is a d-dimensional index, $\mathrm{k}_{\mathrm{i}} \hat{\imath} ¥(\mathrm{i}=1, \mathrm{~L}, \mathrm{~d})$ and $|k|=k_{1}+k_{2}+L+k_{d}$. The orthogonal conditions of $\left\{F_{k}(Z)\right\}|k|=0$ are:

$$
\mathrm{E}_{\text {辖 } ;}(\boldsymbol{Z}) \mathrm{F}_{\mathrm{k}}(\boldsymbol{Z})=g_{\mathrm{j}} d_{\mathrm{j} k}
$$




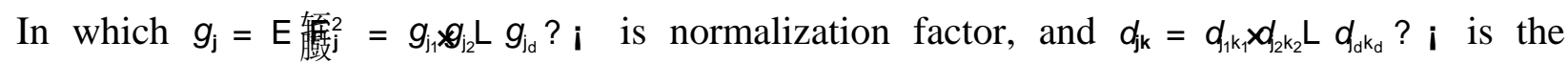
Kronecker function of d-dimension.

In application, proper gPC basis function should be chosen according to distribution type of $z$ to realize optimal convergence rate. Readers are recommended to refer to the literature [6] to know the corresponding relation between random variables and the gPC basis function.

\subsection{The gPC expansion}

The orthogonal conditions ensure that the gPC basis function is an orthogonal basis within the definition domain of $\boldsymbol{Z}$. As a result, the approximate expansion of the observation $\boldsymbol{Y}$ in terms of $\left\{F_{k}(Z)\right\}_{|k|=0}^{N}$ is:

$$
Y(t, X, Z)=\stackrel{\circ}{\mathrm{k}}_{\mathrm{c}_{\mathrm{k}}}(t, X) \mathrm{F}_{\mathrm{k}}(Z)
$$

In which

$$
c_{\mathrm{k}}=\frac{1}{\boldsymbol{g}_{\mathrm{k}}} \mathrm{O}^{Y}(t, X, \boldsymbol{Z}) \mathrm{F}_{\mathrm{k}}(\boldsymbol{Z}) \mathrm{d} \boldsymbol{Z}
$$

$\mathbf{c}_{\mathrm{k}}$ is called the expansion coefficient of gPC and on the same dimension with the observation $Y$. However, since the unknown $Y$ exists on the both sides of the equation, there is no direct way to solve the equation. In this case, the solution of $\mathbf{c}_{\mathrm{k}}$ should go first. Currently, there are two methods to reach the goal: one is the stochastic galerkin method and the other is stochastic collocation method [7]. In the application of stochastic galerkin method, researchers need to derive the random differential equations which can be difficult when the system model is complex. So this paper will focus on the stochastic collocation method in the following section.

\subsection{The Stochastic Collocation Method of gPC}

The biggest difficulty in solving $\mathbf{c}_{\mathrm{k}}$ is to calculate the integral terms in the equation (14) because of $\boldsymbol{Z}$ is still unknown. To explain the basic principles of the Stochastic Collocation Method, we'd better briefly review the Gaussian numerical integration for univariate function. Considering the following integration problem:

$$
\mathrm{I}=\text { Q̀ } f(x) \mathrm{d} x
$$

According to the method of Gaussian numerical integration, the integration of I is approximately equal to:

$$
\text { I 》 } \stackrel{\circ}{\mathrm{k}}_{\mathrm{o}} f\left(x_{\mathrm{k}}\right) w_{\mathrm{k}}
$$

In which $x_{\mathrm{k}}$ is the Gaussian integral point in the definition domain of $x$, and $w_{\mathrm{k}}$ is the corresponding weight of $x_{k}$.Clearly, as long as each $x_{k}$ and $w_{k}$ are known, each $f\left(x_{k}\right) w_{k}$ can be calculated out. Then approximate value of the integration of I. is the sum of every single $f\left(x_{k}\right) w_{k}$.

Similarly, the strategy in realizing the univariate integration can also be applied to approximately solve the integration component in the equation (14). Firstly, $\boldsymbol{Y}(t, \boldsymbol{X}, \boldsymbol{Z}) \mathrm{F}_{\mathrm{k}}(\boldsymbol{Z})$ can be seen as an integrand concerning $\boldsymbol{Z}$. Then, select the collocation points $\boldsymbol{Z}_{\mathrm{j}}$ in the definition domain of $\boldsymbol{Z}$ and calculate its corresponding weight $w_{\mathrm{j}}$. The process of selecting collocation points actually transfer the uncertainty quantity $\boldsymbol{Z}$ to certainty quantity so that the integration part in equation (14) can be carry out by multiplying function values and the corresponding weights. As a result, the equation (14) is approximately equal to:

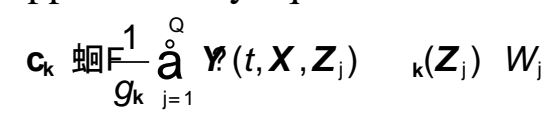

in which $\mathrm{Q}$ refers to the number of collocation points. If $\boldsymbol{z}_{\mathrm{j}}$ and $\boldsymbol{W}_{\mathrm{j}}$ are proper selected, and function expression of $\boldsymbol{Y}(t, \boldsymbol{X}, \boldsymbol{Z})$ and $\mathrm{F}_{\mathrm{k}}(\boldsymbol{Z})$ is known, then $\mathbf{c}_{\mathrm{k}}$ can be approximate calculated by equation(17), so that gPC expansion is solved. 


\section{Simulation analysis}

Assuming that the space station moves around the Earth in a circular equatorial orbit of $500 \mathrm{~km}$ height above the ground from west to east, the duration of redocking is one orbit period $\mathrm{T}$. To improve simulation efficiency, the system governing equation is transformed to dimensionless form, using the average radius of the earth $R_{0}$ as reference length and its corresponding orbit period $T_{0}=2 p \sqrt{R_{0}^{3} / m}$ as reference period, and the dimensionless time is marked as $t$. The pitch angle $q_{1}$ is selected as observation. In the process of redocking, the attitude of the system is not controlled. Take the mass of the space station "Mir" as a reference value [8].

There are two typical initial conditions: (1) at the moment $t_{0}$, the system is at the zero state. That is, the initial attitude angle $\boldsymbol{q}_{0}=\mathbf{0}$ and the angular rate $\boldsymbol{w}_{0}=\mathbf{0}$; (2) at the moment $t_{0}$, the system is at the preceding state. That is, there exists the non-zero initial attitude angle and angular rate $\boldsymbol{w}_{0}$, but the system will return to the zero state when redocking finishes.

In the following parts, this paper will mainly analyze the influence of three kinds of uncertain factors on the pitch angle $q_{1}$ : (1) the uncertainty of cabins' mass; (2) the uncertainty of the mass of center; (3) the uncertainty of the initial attitude angle and angular rate. Suppose all uncertainties are all uniformly distributed, Legendre should be selected as the basis function of gPC according to Table 1 and determine the highest order of the basis function $\mathrm{N}=4$.

\subsection{Uncertainty of cabins' mass}

Suppose that both A and B's mass deviate from nominal value within $\pm 10 \%$. The curves in the upper and lower graphs in Fig. 1 describe the pitch angle in the two kinds of initial conditions. Apparently, the two curves of gPC perfectly overlap with those of the deterministic system, and the standard deviations at the nodes can be barely seen. This means that the deviation of the mass within $\pm 10 \%$ doesn't obviously affect the pitch angle $q_{1}$ in the process of redocking.

ZERO State Redocking

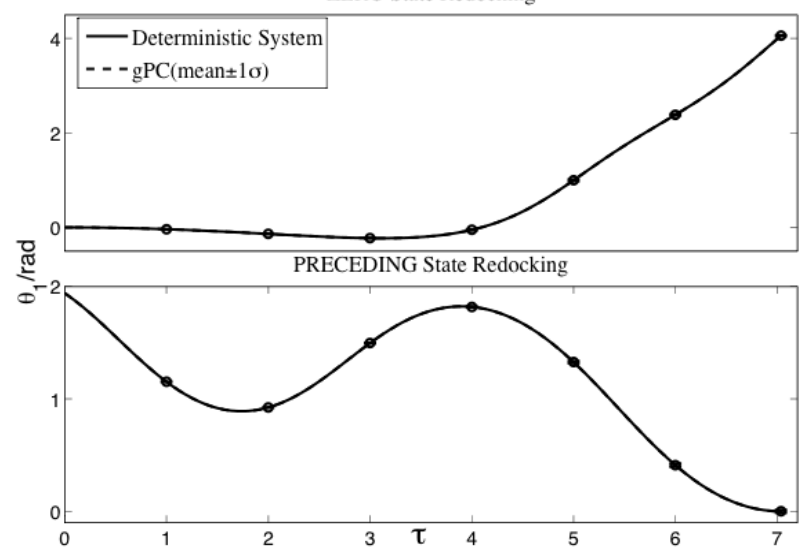

Fig 1. Pitch Angle Curve with Mass Uncertainty

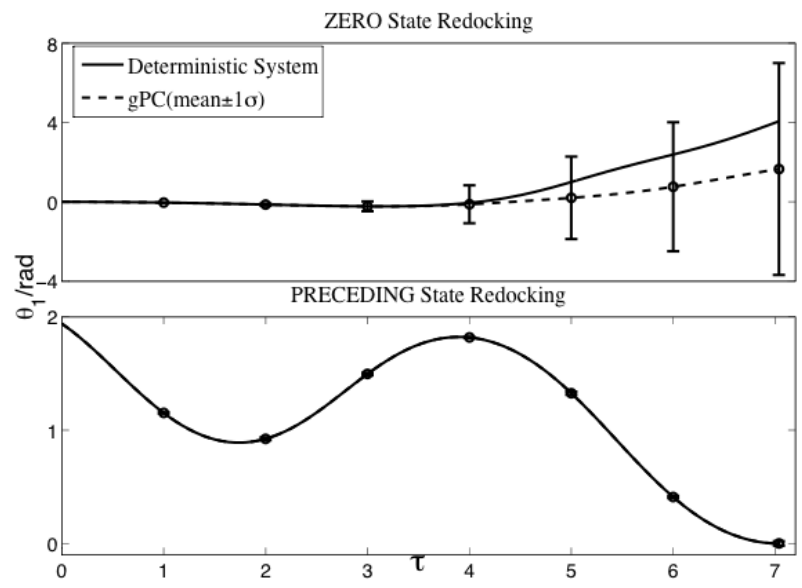

Fig 2. Pitch Angle Curve with C.M. Uncertainty

\subsection{C.M. uncertainty}

Suppose that the deviation between the real C.M. of A and B and the reference C.M. on each dimension is within $\pm 0.1 \mathrm{~m}$. Figure 2 shows the pitch angle curves of redocking in the two kinds of initial conditions. When redocking starts at the zero state, the real curve overlaps with the reference curve before the moment 4 but deviates obviously further and further after. However, when starts at the preceding state, the two curves match very well.

\subsection{Initial state uncertainty}

Suppose there is a deviation of $\pm 1 \mathrm{deg}$ in terms of the initial attitude angle and of $\pm 0.1 \mathrm{deg} / \mathrm{s}$ regarding the initial angular rate. The pitch angle curves with initial state uncertainty are like the curves in the Fig2. Apparently, the curve of redocking from the zero state deviates more and more 
seriously although the deviation at the very beginning is minimal. Nevertheless, the state uncertainty rarely influences the pitch angles if the space station redocks from the preceding state.

\subsection{Cause analysis}

After analyzing the three uncertainty factors, we can conclude that the change of pitch angle is also related to the initial state. The influence of the zero state is obvious, however, the influence of the preceding state is so small that can be neglected. The reason is that the shape of the space station changes during redocking in result of big variation of the system inertial and so attitude motion, both the shape and mass property of the whole space station become asymmetry after redocking.

If the space station starts redocking at the zero state, the induced moment in the result of gravity gradient torque and the system angular rate will become bigger and bigger as the asymmetry of the space station in the process appears more and more obvious. In this case, the initial deviation accumulates and be amplified in a short time so that the attitude angle will deviate further compared with the reference angle value. If the station starts redocking at the preceding state, the preceding angle and its angular rate can be partially cancelled by the attitude angle and its angular rate in the result of gravity gradient torque. Therefore, the preceding angle tends to increase firstly and decrease later. Since the range of the preceding angle changing is slight, the deviation can remain in a low level.

\section{Conclusion}

This paper uses polynomial chaos (gPC) to analyze the influence of the uncertainties on redocking. In addition, mass uncertainty barely affects the changes of attitude angle in the two kinds of initial state. However, even minimal C.M. uncertainty or initial state uncertainty can lead to significant deviation in the end if redocking starts at the zero state. To relieve such situation, it is useful to design the space station to start redock from the preceding state.

\section{References}

[1]. Guangxing Li,Yuzhi Xiao,Shao-Hua Bu,et al. Research on Attitude Control Scheme during Space Station Assembly [J]. Manned Spaceflight. 2012, 18(1): 22-29.

[2]. Yao Y, Guo J, Zhao H, et al. N-body Flexible Dynamics of the Space Station during Module Redocking via Manipulator System: AIAA Guidance, Navigation, and Control Conference, Toronto, Ontario, Canada, 2010[C]. AIAA, August 2-5.

[3]. Yanhong Ma,Jun Zhang,Tingrong Guo. The Attitude Command Optimization during Space Station Assembly [J]. Manned Spaceflight. 2010, 16(1): 17-20.

[4]. Xiu D. Numerical Methods for Stochastic Computations: A Spectral Method Approach [M]. Princeton, New Jersey: Princeton University Press, 2010.

[5]. Rui Chen, Shengjing Tang, Guojiang Sun. Simulation and Analysis of Space Station Redocking[C]. Danang, Vietnam: Springer Verlag, 2014.

[6]. Xiu D, Karniadakis G E. The Wiener-Askey Polynomial Chaos for Stochastic Differential Equations [J]. SIAM J. Sci. Comput., 2002, 24(2):619-644.

[7]. Xiu D. Efficient Collocational Approach for Parametric Uncertainty Analysis [J]. Communications in Computational Physics, 2007, 2(2):293-309.

[8]. Fehse W. Automated Rendezvous and Docking of Spacecraft [M]. Cambridge University Press, 2003. 